These indicators were used in a new concept of training of prehospital command and control. The performance indicators were emphasized to students in lecture form and in different simulation sessions. In the practical exam, these indicators were used as a quality tool for passing or failing the students. In 15 months, this new concept of training of medical first responders has spread to over 750 users in Sweden. Conclusion: The postulated set of performance indicators could be used in any training of medical first responders at the scene of a major incident or disaster. Results can be presented numerically to serve as a quality measurement tool.

Keywords: command and control; first responders; performance indicators; training

Prehosp Disast Med 2005;20(2):s53-s54

\section{Bioterrorism Training and Curriculum Development Program}

Sharon K. Mailey

Shenandoah University, Winchester, Virginia USA

Collaborative Partners: School of Pharmacy, Division of Nursing \& Division of Physician Assistant Studies

There is a compelling need for clinicians to be trained in recognizing and responding to a bioterrorist event, creating an urgency to revisit the planning and educational processes to address the necessary changes. This project demonstrates a model to bridge the curriculum gap of knowledge, skills, and experiences for bioterrorism preparedness in three graduate, healthcare, clinician groups: (1) pharmacy; (2) physician assistant (PA); and (3) advanced-practice nurses (APN). To ensure that an adequate supply of healthcare providers are trained in bioterrorism surveillance and monitoring, Shenandoah University faculty are committed to developing and implementing curricular materials to teach the fundamentals of bioterrorism event recognition, documentation, monitoring of events, and the therapeutic management of patients who are affected.

The curricular content required to address these gaps utilizes online technology, personal digital assistants (PDA), and experiential components that include a multitiered drill. The project involves a student cohort of approximately 160 students including: (1) 109 second-year pharmacists; (2) 37 PAs; and (3) 13 APNs. The cohort provides for a systems theory model approach, which is applied to curriculum design and evaluation, and which emphasizes input (knowledge and skills), process (experiential component), and output (performance-based, multitiered, multi-disciplinary, bioterrorism exercise).

Decision-making is the key element in training for a disaster. In order to develop information-tracking proficiencies and data-acquisition skills for patient management, students will be provided with a PDA programmed with preconfigured software. A database interface developed at Shenandoah University will be used, which consists of a computational algorithm that spawns e-mail if/when critical numbers of symptoms appear. This database will consolidate patient information from students to demonstrate epidemiological principles including recognition of increased incidence patterns of symptom/disease complexes. The clinical preceptors will be oriented to the project and given access to the online bioterrorist course, thereby cascading the training of clinicians. Decision-making will be evaluated using the performance-based bioterrorism exercise with the Winchester Medical Center in Winchester, Virginia.

Keywords: advanced-practices nurse (APN); bioterrorism; curriculum; disaster; education; personal digital assistant; pharmacists; physician assistant (PA); preparedness; training Prehosp Disast Med 2005;20(2)::54

Education and Training for Mass-Casualty Incidents: A One-Day Concept Combining Theoretical Background, Interactive Workshops, and Hands-On Training in a Real-Time Simulation

K. Wilhelm; F. William; C. Günzel; H. Estner

Förderverein Rettungsdienst Dachauer Land e.V., Germany

As the possibility of mass-casualty incidents (MCI) in modern industrial countries due to public transportation crashes and terrorist attacks has increased over recent years, we must ask whether we are prepared for these incidents. Emergency medical services (EMS), fire brigades, and local authorities need background information, detailed plans for action, and actual practice together to make sure their plans are efficient.

An educational concept was developed, combining these three aims to give an update on current strategies. The result was a one-day course on 11 September 2004 in Dachau. It consisted of: (1) presentations of the theoretical backgrounds by international specialists; (2) interactive workshops under the guidance of these specialists; and (3) a live scenario in which the participants were able to put the developed concepts to the test.

The theoretical presentations covered topics such as terrorist attacks on public buses in Israel, management of a major railway crash in Eschede/Germany, international programs for airplane crashes from the United States (US), and a presentation about an airplane collision over southern Germany. The presenters were international specialists from Germany, Israel, and the US, all of whom had not only the theoretical background, but also practical experience with these topics.

In the second part of the day, the participants were assigned to nine different workshops according to their actual function within the rescue system, e.g., fire chiefs, incident commanders, coordinating emergency physicians (CEP), members of the rescue coordination center (RCC), etc. These workshops were guided by international and local experts to develop modern concepts that fit into the actual local settings. The aim was that, by the end of the day, every workshop should be able to present a working strategy for a simulated mass-casualty incident.

The results of the workshops then were tested in simulated $\mathrm{MCI}$ in which a public bus with $>50$ passengers was hit by a car carrying a bomb inside. Together with the local EMS and fire brigade of Dachau, the participants tried to respond according to the plans developed in the workshops to manage this MCI.

With more than 250 participants from Germany, Austria, and Switzerland, this course was the source of a 